\title{
99mTc-IgG-Lung Scintigraphy in the Assessment of Pulmonary Involvement in Interstitial Lung Disease and Its Comparison With Pulmonary Function Tests and High-Resolution Computed Tomography: A Preliminary Study
}

\author{
Mehrzad Bahtouee ${ }^{1}$; Jamshid Saberifard ${ }^{2}$; Hamid Javadi ${ }^{3}$; Iraj Nabipour ${ }^{4}$; Hasan \\ Malakizadeh ${ }^{1}$; Gholamhossein Monavvarsadegh ${ }^{1}$; Hoda Ilkhani Pak ${ }^{5}$; Azadeh Sadeghi ${ }^{5}$; \\ Majid Assadi ${ }^{5, *}$ \\ ${ }_{2}^{1}$ Department of Internal Medicine (Division of Pulmonary), Bushehr Medical Center Hospital, Bushehr University of Medical Sciences, Bushehr, Iran \\ ${ }_{3}^{2}$ Department of Radiology, Bushehr Medical Center Hospital, Bushehr University of Medical Sciences, Bushehr, Iran \\ ${ }_{3}^{3}$ Research Center of Gastroenterology and Hepatology (GRCGH), Golestan University of Medical Sciences, Gorgan, Iran \\ ${ }_{5}^{4}$ The Persian Gulf Tropical Medicine Research Center, Bushehr University of Medical Sciences, Bushehr, Iran \\ ${ }^{5}$ The Persian Gulf Nuclear Medicine Research Center, Bushehr University of Medical Sciences, Bushehr, Iran \\ ${ }^{*}$ Corresponding author: Majid Assadi, The Persian Gulf Nuclear Medicine Research Center, Bushehr University of Medical Sciences, Bushehr, Iran. Tel:+98-7712541828, E-mail:assadipoya@yahoo.com
}

Received: September 3, 2013; Revised: January 14, 2014; Accepted: March 29, 2014

\begin{abstract}
Background: The discrimination of inactive inflammatory processes from the active form of the disease is of great importance in the management of interstitial lung disease (ILD).

Objectives: The aim of this study was to determine the efficacy of $99 \mathrm{mTc}-\operatorname{IgG}$ scan for the detection of severity of disease compared to highresolution computed tomography (HRCT) and pulmonary function test(PFT).

Patients and Methods: Eight known cases of ILD including four cases of Mustard gas (MG) intoxication and four patients with ILD of unknown cause were included in this study. A population of six patients without lung disease was considered as the control group. The patients underwent PFT and high-resolution computed tomography scan, followed by $99 \mathrm{mTc}-\mathrm{IgG}$ scan. They were followed up for one year. 99mTc-IgG scan assessment of IgG uptake was accomplished both qualitatively (subjectively) and semiquantitatively.

Results: All eight ILD patients demonstrated a strong increase in 99mTc-IgG uptake in the lungs, compared to the control patients. The 99mTc-IgG scan scores were higher in the patient group $(0.64[95 \%$ confidence interval $(\mathrm{CI})=0.61-0.69]))$ than the control group (0.35 $(0.35[95 \% \mathrm{CI}=0.28-0.40]),(\mathrm{P}<0.05))$. In patients, a statistically significant positive correlation was detected between $99 \mathrm{mTc}$-IgG scan and HRCT scores (Spearman's correlation coefficient $=0.92, \mathrm{P}<0.008$ ). The $99 \mathrm{mTc}$-Human Immunoglobulin $(\mathrm{HIG})$ scores were not significantly correlated with PFT findings (including FVC, FEV1, FEV1/FVC), $\mathrm{O}_{2}$ saturation and age ( $\mathrm{P}$ values $>0.05$ ). There were no significant correlations between 99mTc-IgG score and HRCT patterns including ground glass opacity, reticular fibrosis and honeycombing $(\mathrm{P}$ value $>0.05)$.

Conclusion: The present results confirmed that 99mTc-IgG scan could be applied to detect the severity of pulmonary involvement, which was well correlated with HRCT findings. This data also showed that the 99mTc-IgG scan might be used as a complement to HRCT in the functional evaluation of the clinical status in ILD; however, further studies are recommended.
\end{abstract}

Keywords: Interstitial Lung Disease; Tomography, X-Ray Computed; Respiratory Function Tests; Mustard Gas

\section{Background}

Interstitial lung disease (ILD) and diffuse parenchymal lung disease describe a varied range of inflammation and fibrosis in the alveoli, distal airways, and septal interstitium of the lungs (1). Rapid and accurate diagnosis of ILD can be challenging,yet it is critical for patient outcome(1). Clinical diagnosis is based on the integration of clinical, radiological and histopathological features (2). The ILD is recognized by lung biopsy, which is an invasive modality, and is difficult to repeat during follow-up (2). Moreover, histological findings are not always in accordance with the clinical severity of the disease (3).

High-resolution computed tomography (HRCT) and pulmonary function tests (PFTs) are the most frequently used noninvasive modalities for the evaluation of interstitial lung disease (4). HRCT is considered to be the "gold standard", as its findings are meticulously related to histological findings (5). Nevertheless, the clinical efficacy of this technique is influenced by the qualitative interpretation of the results (5). However, when the findings of PFTs and HRCT are equivocal or conflicting, their clinical value is questionable (6). Therefore, the ideal approach to study pulmonary involvement in patients connective tissue disease (CTD) has still not been determined.

In this respect, a number of radiopharmaceuticals such as 67 Ga-citrate and 111 In-octreotide in the lungs and diminished clearance time of 99mTc-DTPA aerosol for scin-

Copyright ( 2015, Tehran University of Medical Sciences and Iranian Society of Radiology. This is an open-access article distributed under the terms of the Creative Commons Attribution-NonCommercial 4.0 International License (http://creativecommons.org/licenses/by-nc/4.0/) which permits copy and redistribute the material just in noncommercial usages, provided the original work is properly cited. 
tigraphic recognition of ILD have been suggested (7), yet an optimal agent with high concordance with HRCT and PFTs is still missing (8).

Technetium (99mTc)-labeled polyclonal Immunoglobulin $\mathrm{G}(\operatorname{Ig} G)$ is a radiopharmaceutical, which is extensively applied for the detection of focal or diffuse infection and inflammation (9). Although results from limited preclinical and pilot studies in patients are promising (10), this important agent is not currently available for routine clinical use.

\section{Objectives}

The aim of this study was to assess the efficacy of 99mTcIgG lung scan in evaluation of the severity of pulmonary involvement in patients with ILD.

\section{Patients and Methods}

This study was conducted on eight patients who were referred from the lung department to our nuclear medicine center with a history of interstitial lung disease, due to Mustard gas intoxication and/or unknown cause. Patients with a history of chronic respiratory disease, heart failure, lung cancer, contemporary history of respiratory infection at the time of investigation, severe pulmonary arterial hypertension (PAH), Ig-A deficiency, and history of immune reaction to blood transfusion were excluded. Patients were recruited from a university hospital from 2011 to 2013. The patients underwent PFT and HRCT, followed by $99 \mathrm{mTc}$-IgG scan. They were followed for one year. A population of six patients without lung disease who were evaluated with 99mTc-human immunoglobulin (HIG) scintigraphy for the diagnostic workup of osteomyelitis was also included as control patients for 99mTcIgG uptake in the lungs.

The study complied with the declaration of Helsinki and was approved by institutional ethics committee of Bushehr university of medical sciences and all patients provided written informed consent.

\subsection{Severity Grading of Respiratory Complications}

Pulmonary function variables were measured, using a spirometer (ZAN 100, Me, grerate GmbH, Germany). The severity grading of respiratory impairment was based on the values of patient's Forced Vital Capacity (FVC) and Forced Expiratory Volume during the First Second (FEV1) (10).

\subsection{High-Resolution Computed Tomography Scan}

HRCT images were performed on an electron beam CT machine. All $1.5 \mathrm{~mm}$ sections were obtained at full inspiration, both in supine and in prone position. In all cases, additional expiratory films and prone sections were taken. Images were photographed with window settings appropriate for viewing lung parenchyma. The scans, together with plain chest radiographs, were assessed by a pulmonary radiologist. The presence or absence of patchy ground glass pattern, reticular pattern, emphysema, honeycombing, airways distortion, and bronchial wall thickening scans were recorded (7). The parenchymal abnormalities identified on HRCT were coded, and a score was defined according to Goldin et al. (11). For the appraisal of the severity of disease, a point value was allocated to each abnormality as follows: pure ground glass opacity, fibrosis (including thickened reticular markings, bronchiectasis and bronchiolectasis) and honeycombing (11).

An "extent of disease" score was acquired by the percentage of disease extent for each abnormality: normal was scored 0, <25\%; 1, 25 - 50\%; 2, 51 - 75\%; 3 and > 75\%: 4. Three zones were considered as follows: zone 1, apex to aortic arch; zone 2, aortic arch to inferior pulmonary veins; and zone 3, inferior pulmonary veins to diaphragms; right and left lung were scored separately (11). Finally, the scores of severity and extent of disease were added to form the total HRCT score (11).

\subsection{Imaging Protocols}

\subsection{1. $99 m T c-I g G$ Scintigraphy}

Scans were performed using a dual head ADAC camera, (ADAC Genesys Malpitas, CA, USA) equipped with a pair of low energy, high-resolution collimators. For 99mTc-IgG Scintigraphy, a human nonspecific polyclonal immunoglobulin (IVIG) for intravenous administration was used. IgG solution (10 mg/mL in 0.01 M PBS buffer, $\mathrm{pH} 7.4$ ) was used to prepare $99 \mathrm{mTc}-\operatorname{IgG}$ by adding $740 \mathrm{MBq}(20 \mathrm{mCi})$ to $99 \mathrm{~m}$-Tc followed by incubation for 20 minutes at room temperature. IgG kit was provided by the nuclear energy institute of Iran. Furthermore, 99mTc-IgG was administered intravenously and early (two hours) and delayed (four hours) imaging was performed, for at least 400000 counts. Anterior and posterior views of the thorax were obtained during each phase of the study.

\subsubsection{Image Analysis}

Images were assessed by two nuclear medicine physicians, who were blinded to all other clinical and imaging information. Interobserver variability was resolved by consensus. Patients showing increased uptake in early or delayed or both images were regarded as positive. The radionuclide uptake was characterized by: No uptake (-); mild uptake $( \pm)$ moderate $(+)$ and severe uptake $(++)$.

For semiquantitative analysis, region of interest (ROI) analysis was carried out on the anterior and posterior aspects. ROIs of the same size were drawn over the upper, middle, and lower regions of each lung (12). The ratio of the mean counts of these ROIs and the counts of a ROI over the left ventricle divided by two gave a value for each patient, which represented the disease severity using 99mTc-HIG scintigraphy (HIG score) (12). 


\subsection{Statistical Analysis}

The Man-Whitney U test was applied for the quantitative comparison of HIG scores between patients and controls groups. Spearman's correlation coefficient was used for correlations. A p value less than 0.05 was considered statistically significant.

\section{Results}

This study included seven males and seven females (mean age, $53.58 \pm 7.60$; range, 43 - 70 years). Eight patients had interstitial lung disease, four had a history of Mustard gas exposure and the remaining four patients didn't have any known cause (ILD of unknown cause). The study population also included six patients who had referred to the center for evaluation of infection process of limbs. None had a history of suspected or documented lung abnormality.

None of the patients had any adverse reactions after intravenous injection of 99mTc-IgG and no side effects were reported up to 14 days following the administration.

Qualitative analysis of 99mTc-IgG scan in the six control patients showed no significant uptake in the lungs. Four patients showed severe activity on the 99mTc-IgG scan and four patients showed mild activity (Figure 1).

All eight ILD patients demonstrated a strong increase in 99mTc-IgG uptake in the lungs, compared to the control group. Scores of $99 \mathrm{mTc}$-IgG scan were higher in the patient group (0.64[95\% confidence interval $(\mathrm{CI})=0.61-0.69])$ than the control group $(0.35[95 \% \mathrm{CI}=0.28-0.40]))(\mathrm{P}<0.05)$. A positive correlation was detected between 99mTc-IgG scan and HRCT scores in patients (Spearman's correlation coefficient $=$ $0.92, \mathrm{P}<0.008)$.

PFT grading showed that four patients were in grade 4 (severe) and four were in grade 1 (normal). Scores of 99mTc-IgG scan was not significantly correlated with PFT findings (including FVC, FEV1, FEV1/FVC), $\mathrm{O}_{2}$ saturation and age ( $P$ values $>0.05$ ). Pulmonary involvement was not concordant between HRCT and 99mTc-IgG scan. For example, one patient with HRCT score of 14, had intense activity on 99mTc-IgG scan, while another with HRCT score of 16, had mild activity on 99mTc-IgG scan.

The association between $99 \mathrm{mTc}$-IgG scan scores and HRCT patterns including ground glass opacity, reticular fibrosis and honeycombing were not statistically significant (P value $>0.05$ ). In addition, we did not observe a significant association between 99mTc-IgG scan score and HRCT scores in three classified zones (P value $>0.05$ ).

The 99mTc-IgG scan score was higher in patients with Mustard gas exposure than patients with unknown ILD $(P=0.04)$. The 99mTc-IgG scan scores were not different according to the outcome after the one-year follow up (dead or alive; P value $>0.05$ ). It should be noted that the patients who died during the one-year follow up were older than the remaining cases $(\mathrm{P}<0.05)$.
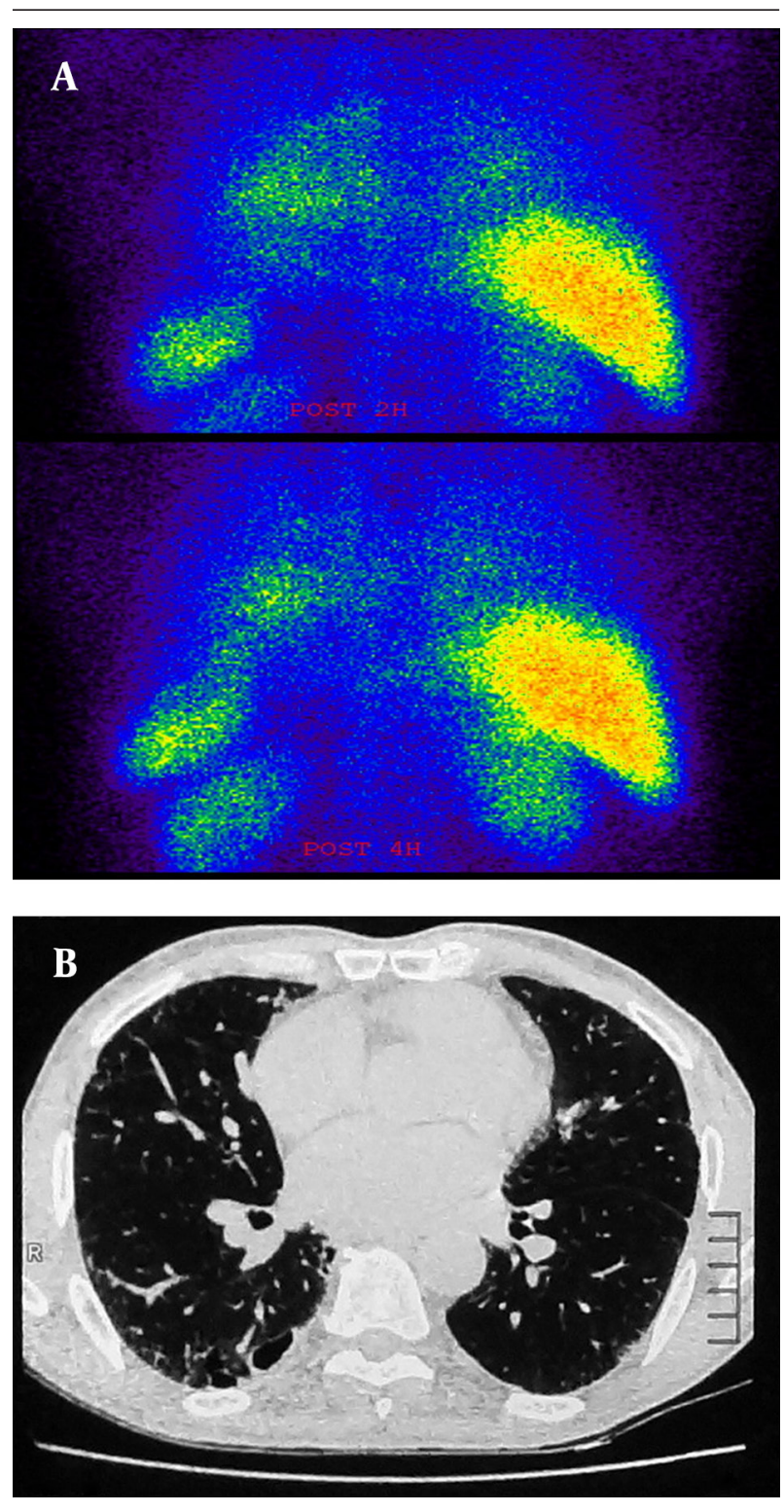

Figure 1. A, there was significant activity in the lung fields in the early views (upper row) of 99mTc-IgG scintigraphy of a 56-year-old man, which persisted over the delayed views for up to four hours (lower row). The early HIG score was 0.85 and the delayed HIG score was 0.91. The images are in the posterior projection. B, HRCT scan of the same patient showing some degree of interstitial fibrosis and ground glass opacities throughout the lungs (score 14).

\section{Discussion}

This study highlights the advantages of 99mTc-IgG imaging as a rapid and accurate imaging technique for the evaluation of inflammatory processes in the lungs. Although there was an association between $99 \mathrm{mTc}-\mathrm{IgG}$ and HRCT scores, there were a few cases of higher 99mTc-IgG scores in the lower HRCT group compared to the higher HRCT group. This might imply that nuclear imaging modalities can easily detect an inflammatory process because they are based on functional processes (13). 
The $99 \mathrm{mTc}$-IgG is a radiotracer that has been applied in studies of focal infection and inflammation in immune disorders such as rheumatoid arthritis (RA), Sjogren's syndrome and inflammatory bowel disease $(7,9,14,15)$. Numerous uptake mechanisms have been illustrated to contribute to $99 \mathrm{mTc}-\operatorname{IgG}$ accumulation $(7,15)$. Primarily, 99mTc-IgG uptake thought to be due to increased vascularity and vascular permeability at the site of inflammation.

In the current study, there was no association between 99mTc-IgG scores and PFT results, which may indicate the importance and necessity of 99mTc-IgG in the evaluation of the severity of pulmonary involvement. It has been reported that 111 In-octreotide uptake in the lung is correlated with the fibrosis score in HRCT but not with the ground-glass score in the present idiopathic pulmonary fibrosis patients (16). In our study, there was no association between the patterns of HRCT and HIG scores. This may be due to different populations and low numbers of patients included in this study (16).

We found that the 99mTc-IgG scores were higher in Mustard gas injuries compared to unknown ILD patients. Inhalation of sulfur mustard mainly affects the upper respiratory tract, and there is no definite nature of lung pathology in patients whom are exposed to sulfur mustard (SM) many years after exposure. However, the most common inflammatory cells in bronchoalveolar lavage (BAL) fluid were shown to be neutrophils (88\%) (17).

The 99mTc-IgG scores were not significantly different in alive and dead patients during follow-up, however the patients who died during the follow up were older than the other cases.

Among radiopharmaceuticals, 99mTc-IgG scans have shown the most promising results for the differentiation of active and inactive lesions in limited human studies (18).

To our knowledge, this is the first clinical study to compare 99mTc-IgG scan with conventional modalities for the detection of inflammation in injured patients with Mustard gas. In a limited number of studies the role of 99mTc-IgG scan in the assessment of pulmonary involvement was assessed $(12,19)$.

Koutsikos et al. investigated the value of $99 \mathrm{mTc}$-HIG lung scintigraphy for the diagnosis of pulmonay inflammation (PI) in 16 connective tissue disease (CTD) patients with mild HRCT abnormalities (19). In the total population, they reported a statistically significant positive correlation between 99mTc-IgG scan and HRCT scores ( $\mathrm{r}$ $=0.622, \mathrm{P}<0.001)$, and a negative correlation between 99mTc-IgG scan scores and diffusing capacity of the lungs for carbon monoxide (DLCO) $(r=-0.203, P>0.05)$, and HRCT scores and DLCO $(r=-0.009, \mathrm{P}>0.05)$. Furthermore, they found that $99 \mathrm{mTc}-\mathrm{IgG}$ scan scores were higher in group A (12 patients with pulmonary involvement) ( 0.58 \pm 0.05 ) than in group B (4 patients without pulmonary involvement $)(0.5 \pm 0.09)(\mathrm{P}<0.05)$. They concluded that 99mTc-IgG scan seems to be more accurate than HRCT in the discrimination of affected patients (19).

The efficacy of 99mTc-IgG lung scan in assessment of the severity of pulmonary involvement was also evaluated in CTD patients (12). A Significantly higher 99mTc-IgG scan score was reported in patients with CTD $(0.6 \pm 0.07$ vs. $0.51 \pm 0.08)$. Moreover, there was a negative correlation between $99 \mathrm{mTc}-\mathrm{IgG}$ scan scores and PFT results as well as a positive correlation between 99mTc-IgG scan and HRCT scores. The 99mTc-IgG scan has demonstrated similar clinical efficacy as HRCT, with higher sensitivity (77.5 vs. 57.5\%) but lower specificity (75 vs. 91.7\%) (12).

In the present study, we found differences between the inflammation indices of patients and control groups for the 99 mTc-IgG scan performed at one hour and four hours, therefore it seems that the one hour scan is sufficient and delayed scintigraphy is not required.

Although our study demonstrates good insight into using 99mTc-IgG as compared with clinical and radiological assessment, it should be noted that these results have some shortcomings. The major limitations were the small sample size and the absence of lung biopsy for all subjects as a gold standard test, both of which may have influenced the results of this study; however, we did consider compound clinical presentation, radiological examinations, and follow-up evaluation to mitigate this deficiency. Our results should be validated in a larger and well-designed study.

In conclusion, the present preliminary results confirmed that 99mTc-IgG scan can be applied to detect the severity of pulmonary involvement in early views, which was well correlated with HRCT findings. These data also showed that the use of 99mTc-IgG in lung studies might be used as a complement to HRCT in the functional evaluation of the clinical status in ILD.

\section{Acknowledgements}

We would like to express our thanks to our colleagues, especially SR. Mosavi, M. Kassaian and Kh. Golzari, for their assistance in this research.

\section{Funding/Support}

This study was sponsored by Bushehr University of Medical Sciences (grant no. 546).

\section{References}

1. Latsi PI, Wells AU. Evaluation and management of alveolitis and interstitial lung disease in scleroderma. Curr Opin Rheumatol. 2003;15(6):748-55.

2. Flaherty KR, King TE, Jr, Raghu G, Lynch JP, 3rd, Colby TV, Travis WD, et al. Idiopathic interstitial pneumonia: what is the effect of a multidisciplinary approach to diagnosis? Am J Respir Crit Care Med. 2004;170(8):904-10.

3. Bouros D, Wells AU, Nicholson AG, Colby TV, Polychronopoulos V, Pantelidis P, et al. Histopathologic subsets of fibrosing alveolitis in patients with systemic sclerosis and their relationship to outcome. Am J Respir Crit Care Med. 2002;165(12):1581-6.

4. Lynch DA, Godwin JD, Safrin S, Starko KM, Hormel P, Brown KK, et al. High-resolution computed tomography in idiopathic pulmonary fibrosis: diagnosis and prognosis. Am J Respir Crit Care Med. 2005;172(4):488-93. 
5. Wells AU, Hansell DM, Corrin B, Harrison NK, Goldstraw P, Black $\mathrm{CM}$, et al. High resolution computed tomography as a predictor of lung histology in systemic sclerosis. Thorax. 1992;47(9):738-42.

6. Afeltra A, Zennaro D, Garzia P, Gigante A, Vadacca M, Ruggiero A, et al. Prevalence of interstitial lung involvement in patients with connective tissue diseases assessed with high-resolution computed tomography. Scand J Rheumatol. 2006;35(5):388-94.

7. Asli IN, Javadi H, Seddigh H, Mogharrabi M, Hooman A, Ansari M, et al. The diagnostic value of (99m)Tc-IgG scintigraphy in the diabetic foot and comparison with (99m)Tc-MDP scintigraphy.J Nucl Med Technol. 2011;39(3):226-30.

8. Mogulkoc N, Brutsche MH, Bishop PW, Murby B, Greaves MS, Horrocks AW, et al. Pulmonary (99m)Tc-DTPA aerosol clearance and survival in usual interstitial pneumonia (UIP). Thorax. 2001;56(12):916-23.

9. Buscombe JR, Lui D, Ensing G, de Jong R, Ell PJ. 99mTc-human immunoglobulin (HIG)--first results of a new agent for the localization of infection and inflammation. Eur J Nucl Med. 1990;16(810):649-55.

10. Etezad-Razavi M, Mahmoudi M, Hefazi M, Balali-Mood M. Delayed ocular complications of mustard gas poisoning and the relationship with respiratory and cutaneous complications. Clin Experiment Ophthalmol. 2006;34(4):342-6.

11. Goldin JG, Lynch DA, Strollo DC, Suh RD, Schraufnagel DE, Clements PJ, et al. High-resolution CT scan findings in patients with symptomatic scleroderma-related interstitial lung disease. Chest. 2008;134(2):358-67.

12. Kostopoulos C, Koutsikos J, Toubanakis C, Moulopoulos LA, Mamoulakis C, Gialafos E, et al. Lung scintigraphy with nonspecific human immunoglobulin G ((99m)Tc-HIG) in the evaluation of pulmonary involvement in connective tissue diseases: correlation with pulmonary function tests (PFTs) and high-resolution computed tomography (HRCT). Eur J Nucl Med Mol Imaging. 2008;35(2):343-51

13. Lupetti A, Welling MM, Pauwels EK, Nibbering PH. Radiolabelled antimicrobial peptides for infection detection. Lancet Infect Dis. 2003;3(4):223-9.

14. de Bois MH, Arndt JW, van der Velde EA, van der Lubbe PA, Westedt ML, Pauwels EK, et al. 99mTc human immunoglobulin scintigraphy--a reliable method to detect joint activity in rheumatoid arthritis. J Rheumatol.1992;19(9):1371-6.

15. Karanikas G, Bobacz K, Becherer A, Wiesner K, Dudczak R, Machold K, et al. Tc-99m-labeled human polyclonal immunoglobulin G (HIG) scintigraphy in Sjogren's syndrome. Scand J Rheumatol. 2002;31(2):80-4.

16. Lebtahi R, Moreau S, Marchand-Adam S, Debray MP, Brauner M, Soler P, et al. Increased uptake of 111In-octreotide in idiopathic pulmonary fibrosis. J Nucl Med. 2006;47(8):1281-7.

17. Emad A, Rezaian GR. The diversity of the effects of sulfur mustard gas inhalation on respiratory system 10 years after a single, heavy exposure: analysis of 197 cases. Chest. 1997;112(3):734-8.

18. Akhtar MS, Qaisar A, Irfanullah J, Iqbal J, Khan B, Jehangir M, et al. Antimicrobial peptide 99mTc-ubiquicidin $29-41$ as human infection-imaging agent: clinical trial. J Nucl Med. 2005; 46(4):567-73.

19. Koutsikos J, Kostopoulos C, Moulopoulos L, Karamini O, Zerva $\mathrm{C}$, Leondi A, editors. HIG scintigraphy in mild HRCT findings in the assessment of pulmonary involvement (PI) associated with connective tissue diseases (CTD).; Society of Nuclear Medicine Annual Meeting Abstracts.; 2008; Soc Nuclear Med; p. 277P. 